New windows on massive stars: asteroseismology, interferometry, and spectropolarimetry

Proceedings IAU Symposium No. 30\%, 2014

G. Meynet, C. Georgy, J. H. Groh \& Ph. Stee, eds.

(C) International Astronomical Union 2015 doi:10.1017/S1743921314006279

\title{
Discovery of a Thorne-Żytkow object candidate in the Small Magellanic Cloud
}

\author{
Emily M. Levesque ${ }^{1}$, Philip Massey ${ }^{2}$, Anna N. Żytkow ${ }^{3}$ and \\ Nidia Morrell ${ }^{4}$ \\ ${ }^{1}$ Center for Astrophysics \& Space Astronomy, University of Colorado UCB 389, Boulder, CO \\ 80309, USA; Hubble Fellow \\ email: Emily.Levesque@colorado.edu \\ ${ }^{2}$ Lowell Observatory, 1400 W. Mars Hill Road, Flagstaff, AZ 86001, USA \\ ${ }^{3}$ Institute of Astronomy, University of Cambridge, Madingley Road, Cambridge CB3 0HA, UK \\ ${ }^{4}$ Las Campanas Observatory, Carnegie Observatories, Casilla 601, La Serena, Chile
}

\begin{abstract}
Thorne-Żytkow objects (TŻOs) are a theoretical class of star in which a compact neutron star is surrounded by a large, diffuse envelope. Supergiant TŻOs are predicted to be almost identical in appearance to red supergiants (RSGs), with their very red colors and cool temperatures placing them at the Hayashi limit on the H-R diagram. The only features that can be used at present to distinguish TŻOs from the general RSG population are the unusually strong heavy-element and lithium lines present in their spectra. These elements are the unique products of the stars fully convective envelope linking the photosphere with the extraordinarily hot burning region in the vicinity of the neutron star core. We have recently discovered a TŻO candidate in the Small Magellanic Cloud. It is the first star to display the distinctive chemical profile of anomalous element enhancements thought to be characteristic of TŻOs; however, upto-date models and additional observable predictions (including potential asteroseismological signatures) are required to solidify this discovery. The definitive detection of a TŻO would provide the first direct evidence for a completely new model of stellar interiors, a theoretically predicted fate for massive binary systems, and never-before-seen nucleosynthesis processes that would offer a new channel for heavy-element and lithium production in our universe.
\end{abstract}

Keywords. stars: peculiar, supergiants, variables

\section{Introduction}

Thorne-Żytkow objects (TŻOs) are a class of star originally proposed by Thorne \& Żytkow $(1975,1977)$, comprised of a neutron star core surrounded by a large and diffuse envelope. They are expected to form as the product of a dual-massive-star binary, with the neutron star forming when the more massive star explodes as a supernova. During subsequent evolution of the system, the expanding envelope of the lower-mass companion may lead to a common envelope state and the spiral-in of the neutron star into the companion's core (Taam et al. 1978). Alternately, Leonard et al. (1994) propose that a TŻO may be produced when a newly-formed neutron star receives a supernova 'kick' velocity in the direction of its red supergiant (RSG) companion and becomes embedded.

TŻOs represent a completely new theoretical class of stellar object, offering a novel model for stellar interiors and an example of unique nucleosynthetic processes. However, there has never been a positive observational identification of a TŻO. They are predicted to be virtually indistinguishable from luminous M-type RSGs, lying at the Hayashi limit (Hayashi \& Hoshi 1961) and showing signs of excess mass loss. To date, the only predicted observational signature of a TŻO is an unusual set of atmospheric chemical abundances, 
produced by the 'interrupted rapid-proton' process or irp-process that is uniquely possible in a stellar interior combining a neutron star core and a completely convective envelope (e.g. Zimmerman 1979; Biehle 1991; Cannon 1993; see also Wallace \& Woosley 1981). Several specific irp-process elements should be observable in a TŻO atmosphere, including lines of Rb I and Mo I (Biehle 1994). ${ }^{7} \mathrm{Li}$ should also be over-abundant in TŻOs due to the ${ }^{7}$ Be-transport mechanism, which is similarly dependent on the star's internal structure (e.g. Cameron 1955; Podsiadlowski et al. 1995). While previous searches have been made for TŻOs, none have observed a star with a chemical profile attributable to a TŻO interior (Vanture et al. 1999; Kuchner et al. 2002).

Here we present our observations of the TŻO candidate HV 2112 in the Small Magellanic Cloud. This star exhibits the distinctive set of anomalous element enhancements thought to be produced by processes unique to TŻOs. We discuss our sample, observations, and analyses (Section 2), discuss the properties of HV 2112 (Section 3), and consider the implications and potential for future work on TŻOs (Section 4).

\section{Observations, Reduction, and Analysis}

We constructed our sample of 22 SMC RSGs from objects observed in our past effective temperature $\left(T_{\text {eff }}\right)$ studies (Levesque et al. 2006) and additional stars with 2MASS photometry and colors consistent with RSGs $(K<8.9, J-K>1)$. Closely-orbiting massive binaries are more common at low metallicities (Linden et al. 2010), making the predicted binary progenitor scenarios for TŻOs more likely in metal-poor host environments such as the $\mathrm{SMC}(\log (\mathrm{O} / \mathrm{H})+12=8.0$, van den Bergh 2000).

Our observations were carried out using the Magellan Inamori Kyocera Echelle (MIKE; Bernstein et al. 2003) and Magellan Echellete (MagE; Marshall et al. 2008) spectrographs on the Magellan 6.5-meter Clay telescope at Las Campanas Observatory on 13-15 Sep 2011. We obtained high-resolution spectra with MIKE (using the $0^{\prime} .7 \times 5^{\prime}$ slit with $2 \times 2$ binning, 'slow' readout, and the standard grating settings to give $R \sim 42,000$ ) for the purpose of measuring line ratios, as well as lower-resolution MagE spectra (using the $1^{\prime \prime}$ with $1 \times 1$ binning and 'slow' readout) for the purpose of producing flux-calibrated spectrophotometry that could be used to determine contemporaneous physical properties for our stars. Our observations were generally performed with seeing $\lesssim 1^{\prime \prime}$ and airmasses $\lesssim 1.5$; our MagE spectra were taken at the parallactic angle, and spectrophotometric standards were observed for flux calibration. The MIKE and MagE data were reduced using standard IRAF echelle routines and the mtools package (see Massey et al. 2012).

To search for signs of anomalous TŻO-like element enhancements in our spectra, we compared the equivalent widths of $\mathrm{Li}, \mathrm{Rb}$, and Mo absorption features - all elements expected to be enhanced in TŻOs - to those of nearby spectral features where no significant enhancements were previously predicted, such as K, Ca, Fe, and Ni (Cannon 1993; Biehle 1994; Podsiadlowski et al. 1995; however, see also Tout et al. 2014). However, line blanketing effects in RSG spectra completely obscure the continuum, rendering traditional measurements of absorption impossible. We therefore adopt the "pseudo-equivalent width" method detailed in Kuchner et al. (2002), and determined pseudo-equivalent width measurements for our lines of interest with systematic errors of $\lesssim 5 \%$.

We calculated the the Rb I $\lambda 7800.23 / \mathrm{Ni}$ I $\lambda 7797.58(\mathrm{Rb} / \mathrm{Ni})$ and $\mathrm{Rb}$ I $\lambda 7800.23 / \mathrm{Fe}$ I $\lambda 7802.47(\mathrm{Rb} / \mathrm{Fe})$ line ratios to probe the relative $\mathrm{Rb}$ enhancement in our stars; the $\mathrm{Li}$ I $\lambda 6707.97 / \mathrm{K}$ I $\lambda 7698.97(\mathrm{Li} / \mathrm{K})$ and $\mathrm{Li} \mathrm{I} \lambda 6707.97 / \mathrm{Ca} \mathrm{I} \lambda 6572.78$ (Li/Ca) ratios for the relative Li enhancement; and the Mo I $\lambda 5570.40 / \mathrm{Fe}$ I $\lambda 5569.62(\mathrm{Mo} / \mathrm{Fe}$ ) ratio for the relative Mo enhancement. To ensure that our ratios were truly probing abundances anomalies in TŻO products rather than variations in our comparison features, we also 

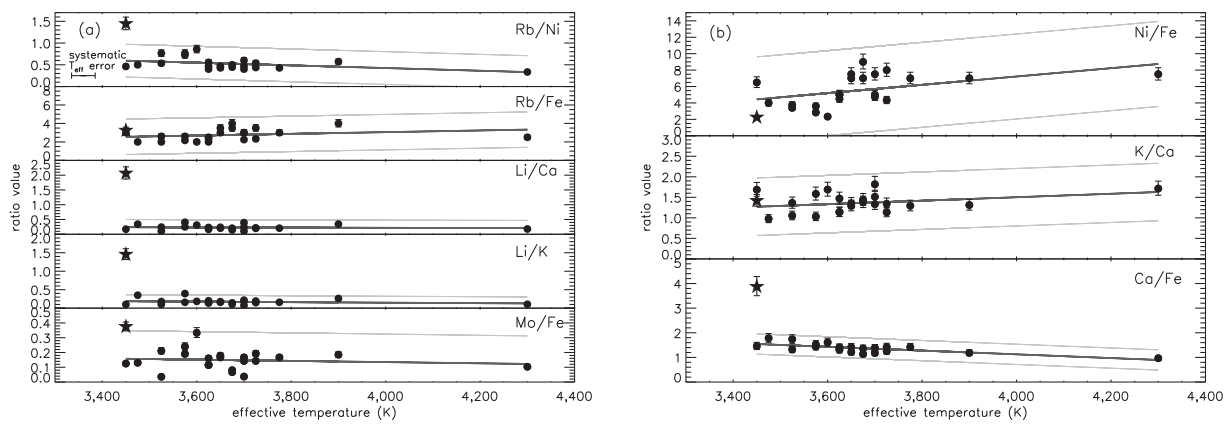

Figure 1. $T_{\text {eff }}$ vs. measured line ratios for our SMC RSGs (circles) and HV 2112 (stars). The ratios include features predicted to be enhanced in TŻOs (a) as well as our 'control' features (b). A dark grey line shows the best linear fit for each line ratio as a function of $T_{\text {eff }}$, while light grey lines mark the $3 \sigma$ deviations from the fit that encompass 99.7 per cent of a normally-distributed sample. Error bars for each point illustrate the systematic errors of $\lesssim 5 \%$.

calculated Ni I $\lambda 7797.58 / \mathrm{Fe}$ I $\lambda 7802.47$ (Ni/Fe), K I $\lambda 7698.97 / \mathrm{Ca}$ I $\lambda 6572.78$ (K/Ca), and $\mathrm{Ca} I \lambda 6572.78 / \mathrm{Fe} \mathrm{I} \lambda 65569.62(\mathrm{Ca} / \mathrm{Fe})$ ratios for our stars.

We compared the line ratios for our stars relative to their measured $T_{\text {eff }}$. We then determined the best linear fit for each set of ratios as a function of $T_{\text {eff }}$, and calculated the $3 \sigma$ variations from these fits that should encompass $99.7 \%$ of a normally-distributed sample. Following this method, we considered a ratio to be statistically anomalous if it fell outside the $3 \sigma$ range. For a more detailed discussion of our observations, reductions, and analysis techniques please see Levesque et al. (2014).

\section{The TŻO Candidate HV 2112}

We found that one star, HV 2112, has anomalously high values of $\mathrm{Rb} / \mathrm{Ni}, \mathrm{Li} / \mathrm{K}, \mathrm{Li} / \mathrm{Ca}$, and $\mathrm{Mo} / \mathrm{Fe}$. The ratios measured for our full sample, including HV 2112, are shown in Figure 1. These ratios present clear evidence of Rb, Li, and Mo enhancement in the star's atmosphere, a combination thought to be possible as a result of the exotic stellar interiors of TŻOs. A comparison of the HV 2112 spectrum to a 'typical' SMC RSG is shown in Figure 2 (left); the HV 2112 spectrum exhibits notably stronger TŻO features. We also measure an atypically high $\mathrm{Ca} / \mathrm{K}$ ratio; recent work suggests that $\mathrm{Ca}$ may be produced in the final stages of TŻO formation (Tout et al. 2014).

Observations of the Ca II triplet in the HV 2112 allowed us to measure a radial velocity (RV) of $\sim 157 \mathrm{~km} \mathrm{~s}^{-1}$, consistent with this star being a member of the SMC rather than a foreground dwarf (Neugent et al. 2010). From fitting a MagE spectrum of HV 2112 with a MARCS stellar atmosphere model (Gustafsson et al. 2008; see Figure 2, right), we determined a $T_{\text {eff }}$ of $3450 \mathrm{~K}$ and a spectral type of M3 I, along with a $V$ magnitude of $13.7 \pm 0.1$. Correcting for the SMC distance modulus of 18.9 (van den Bergh 2000) and

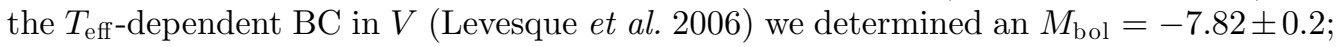
this corresponds to a initial mass of $M \sim 15 M_{\odot}$ (Maeder \& Meynet 2001). We also observed an excess amount of visual extinction in the direction of HV $2112\left(A_{V} \sim 0.4\right.$ as compared to the average $A_{V}=0.24$ for SMC OB stars assuming $R_{V}=3.1$; Cardelli et al. 1989; Massey et al. 1995) and a slight flux excess $\left(\lesssim 10 \operatorname{ergs~s}^{-1} \mathrm{~cm}^{-2} \AA^{-1}\right)$ in the near-UV. Both of these features are thought to be signatures of excess circumstellar dust associated with strong mass loss in RSGs (Levesque et al. 2005; Massey et al. 2005). Finally, past work identified HV 2112 as photometrically and spectroscopically variable (Payne-Gaposchkin \& Gaposchkin 1966; Wood et al. 1983; Reid \& Mould 1990; Smith 

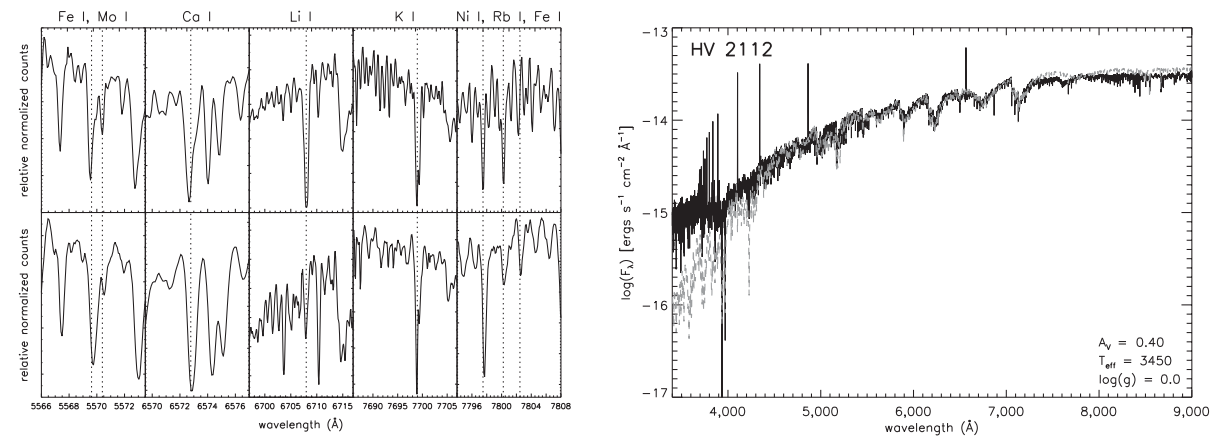

Figure 2. Left: spectral features of HV 2112 (a-e) and [M2002] SMC 005092 (f-j) used in our analyses. [M2002] SMC 005092 is a 'typical' SMC RSG with $T_{\text {eff }}=3475 \mathrm{~K}$, comparable to the $3450 \mathrm{~K} T_{\text {eff }}$ we measure for HV 2112. Spectra are from our MIKE observations and have been corrected to rest-frame. Wavelengths of key absorption features are marked as vertical dashed lines. Right: MagE spectral energy distribution of HV 2112 (solid black line) and the best-fit MARCS stellar atmosphere model (dashed gray). The strong Balmer emission in the HV 2112 spectrum is clearly visible, along with the near-UV excess and near-IR deficiency typical of circumstellar dust effects (Levesque et al. 2005; Massey et al. 2005).

et al. 1995), properties predicted for TŻOs (Thorne \& Żytkow 1977; van Paradijs et al. 1995).

$\mathrm{Rb}$ and Mo have been individually observed in stars and attributed to the $s$-process; however, there are no observed or predicted examples of the $s$-process producing both in a single star along with additional Li enhancement. Simultaneous Rb and Li enhancement is not observed in cool $s$-process stars (e.g. García-Hernández et al. 2013), and the combination of Rb, Mo, and Li has never been observed in any other cool massive star. Futhermore, while $\mathrm{Rb}, \mathrm{Mo}$, and $\mathrm{Li}$ can all individually be produced in super-asymptotic giant branch (SAGB) stars as well as TŻOs (through a combination of the $s$-process and hot bottom burning), an SAGB star cannot synthesize their own Ca and would not produce the Ca enhancement seen in HV 2112 (Tout et al. 2014). Finally, Ba is a common $s$-process product but we see no signs of Ba enhancement in the HV 2112 spectrum (see Vanture et al. 1999). Combined, our observed element enhancements and physical properties of HV 2112 strongly support its classification as a TŻO candidate.

However, HV 2112 does exhibit several unusual spectral features not previously predicted to be associated with TŻOs. The spectrum exhibit strong hydrogen Balmer emission features than extend from $\mathrm{H} \alpha$ out to $\mathrm{H} 18$ (see Figure 2, right) and display a Balmer increment (with $\mathrm{H} \delta$ as the strongest feature followed by $\mathrm{H} \gamma, \mathrm{H} \beta$, and $\mathrm{H} \alpha$ ) similar to that seen in M-type Mira variables. In Miras, this inverted Balmer emission is thought to be produced by non-LTE radiative transfer effects in the hydrogen lines from formation in a shocked atmosphere (Castelaz et al. 2000), with shocks generated by pulsation and propagating through the atmosphere. While the origin of these Balmer emission features in HV 2112 remains unclear, it is likely that the photometric and spectroscopic variability associated with HV 2112 could produce similar pulsationally-driven shocks.

In addition, while the $\mathrm{Rb} / \mathrm{Ni}$ ratio in $\mathrm{HV} 2112$ is far higher than the average ratio measured for SMC RSGs, the Rb/Fe ratio is quite typical. The Ni/Fe ratio in HV 2112 is also typical of the RSG sample, precluding the possible explanation of a Fe overabundance. The Mo, Li, and Rb features do not, at a glance, exhibit the extremely increased strengths one might expect based on the predicted enhancements for TŻOs (Cannon 1993; Biehle 1994; Podsiadlowski et al. 1995); however, relatively weak enhancements could be indicative of an early or short-lived TŻO phase (Thorne \& Żytkow 1975, 1977; 
Podsiadlowski et al. 1995). Convection models for massive star envelopes (particularly at low temperatures) have also advanced significantly in recent years, suggesting that the predicted enhancements for TŻOs could potentially be altered. It is clear that new models of TŻOs are needed; future theoretical work will likely provide updated predictions of element enhancements as well as additional identifying observables that can be used to test whether HV 2112 is indeed a bona fide TŻO.

EML is supported by Hubble Fellowship grant HST-HF-51324.01-A from STScI, which is operated by AURA under NASA contract NAS5-26555. PM acknowledges support from NSF grant AST-1008020. ANŻ thanks the Mitchell Family Foundation for support and Texas A\&M University and Cook's Branch Nature Conservancy for their hospitality.

\section{References}

Bernstein, R., Shectman, S. A., Gunnels, S. M., Mochnacki, S., \& Athey, A. E. 2003, in M. Iye \& A. F. M. Moorwood (eds.), Instrument Design and Performance for Optical/Infrared Ground-based Telescopes, Vol. 4841 of Society of Photo-Optical Instrumentation Engineers (SPIE) Conference Series, pp 1694-1704

Biehle, G. T. 1991, ApJ 380, 167

Biehle, G. T. 1994, ApJ 420, 364

Cameron, A. G. W. 1955, ApJ 121, 144

Cannon, R. C. 1993, MNRAS 263, 817

Cardelli, J. A., Clayton, G. C., \& Mathis, J. S. 1989, ApJ 345, 245

Castelaz, M. W., Luttermoser, D. G., Caton, D. B., \& Piontek, R. A. 2000, AJ 120, 2627

García-Hernández, D. A., Zamora, O., Yagüe, A., et al. 2013, A\& A 555, L3

Gustafsson, B., Edvardsson, B., Eriksson, K., et al. 2008, A\&A 486, 951

Hayashi, C. \& Hoshi, R. 1961, PASJ 13, 442

Kuchner, M. J., Vakil, D., Smith, V. V., et al. 2002, in M. M. Shara (ed.), Stellar Collisions, Mergers and their Consequences, Vol. 263 of Astronomical Society of the Pacific Conference Series, p. 131

Leonard, P. J. T., Hills, J. G., \& Dewey, R. J. 1994, ApJ (Letters) 423, L19

Levesque, E. M., Massey, P., Olsen, K. A. G., et al. 2005, ApJ 628, 973

Levesque, E. M., Massey, P., Olsen, K. A. G., et al. 2006, ApJ 645, 1102

Levesque, E. M., Massey, P., Żytkow, A. N., \& Morrell, N. 2014, MNRAS 443, L94

Linden, T., Kalogera, V., Sepinsky, J. F., et al. 2010, ApJ 725, 1984

Maeder, A. \& Meynet, G. 2001, A\&BA 373, 555

Marshall, J. L., Burles, S., Thompson, I. B., et al. 2008, in Society of Photo-Optical Instrumentation Engineers (SPIE) Conference Series, Vol. 7014 of Society of Photo-Optical Instrumentation Engineers (SPIE) Conference Series

Massey, P., Lang, C. C., Degioia-Eastwood, K., \& Garmany, C. D. 1995, ApJ 438, 188

Massey, P., Morrell, N. I., Neugent, K. F., et al. 2012, ApJ 748, 96

Massey, P., Plez, B., Levesque, E. M., et al. 2005, ApJ 634, 1286

Neugent, K. F., Massey, P., Skiff, B., et al. 2010, ApJ 719, 1784

Payne-Gaposchkin, C. \& Gaposchkin, S. 1966, Smithsonian Contributions to Astrophysics 9, 1

Podsiadlowski, P., Cannon, R. C., \& Rees, M. J. 1995, MNRAS 274, 485

Reid, N. \& Mould, J. 1990, ApJ 360, 490

Smith, V. V., Plez, B., Lambert, D. L., \& Lubowich, D. A. 1995, ApJ 441, 735

Taam, R. E., Bodenheimer, P., \& Ostriker, J. P. 1978, ApJ 222, 269

Thorne, K. S. \& Żytkow, A. N. 1975, ApJ (Letters) 199, L19

Thorne, K. S. \& Żytkow, A. N. 1977, ApJ 212, 832

Tout, C. A., Zytkow, A. N., Church, R. P., \& Lau, H. H. B. 2014, ArXiv e-prints

van den Bergh, S. 2000, The Galaxies of the Local Group, Cambridge 
van Paradijs, J., Spruit, H. C., van Langevelde, H. J., \& Waters, L. B. F. M. 1995, A\&A 303, L25

Vanture, A. D., Zucker, D., \& Wallerstein, G. 1999, ApJ 514, 932

Wallace, R. K. \& Woosley, S. E. 1981, ApJS 45, 389

Wood, P. R., Bessell, M. S., \& Fox, M. W. 1983, ApJ 272, 99

Zimmerman, M. L. 1979, Ph.D. thesis, MASSACHUSETTS INSTITUTE OF TECHNOLOGY.

\section{Discussion}

GREVESSE: Why do you not use spectral synthesis around your lines rather than equivalent widths measured with a "pseudo-continuum"?

LEVESQUE: High-resolution modeling of RSG atmospheres is subject to a number of physical complexities, including treatments of atmospheric geometry, optical depth variations, mass loss effects, and host galaxy abundance variations. To avoid potential biases introduced by the assumptions inherent to atmospheric modeling, we instead simply calculated equivalent width ratios for our spectral lines of interest and compared the resulting ratios across our entire sample to identify outliers. This allowed us to do a direct and consistent comparison that is independent of any modeling assumptions.

VAN BELLE: Why doesn't the formation of a TŻO result in explosive detonation of the system? This seems like the ingredients of a cataclysmic variable.

LEVESQue: This is definitely a good question, and one that we're hoping to explore further in future work with formation models! For now, the Taam et al. (1978) and Leonard et al. (1994) papers detailing the two most commonly-cited massive binary formation mechanisms are probably the best resources for answer this. An interesting consideration along these lines is whether there would be some kind of non-terminal eruptive event or other observable signature during the actual formation process of a TŻO, and this will also hopefully be addressed by future models.

MEYNET: Do we have any information about the rotation rate of the TŻO candidate?

LEVESQUE: We unfortunately don't have estimates of this from our observations, but future work on binary merger models for producing TŻOs would likely be one means of predicting what kind of surface rotation rate we would expect to see.

GROH: Have you had a chance to look at the circumstellar environment of this object, e.g. in the mid-IR? Also, are you able to quantify $\dot{M}$ at this point?

LEVESQUE: We haven't yet examined the circumstellar environment of HV 2112 in the mir-IR, and we can't quantify $\dot{M}$ with our current data. Imaging of the circumstellar environment would certainly be extremely interesting both for studying the mass loss properties and for seeking out any potential remnants of a recent supernova that could have produced the core neutron star.

KHALACK: Have you taken into account the NLTE effects that can contribute to the formation of line profiles that belong to $\mathrm{Li}, \mathrm{Ru}, \mathrm{Mo}$, Y, etc.?

LEVESQUE: This isn't something we have examined directly; our ratio determinations are independent of any modeling assumptions, so NLTE effects are not a primary concern for this work. However, these effects would of course be important in future modeling of TŻO atmospheres! 
KHALACK: How many lines have you used to determine an average ratio of equivalent widths (for two elements)?

LeVEsque: We do not determine an "average ratio" of equivalent widths for two elements; instead, we simply measure the ratio of equivalent widths for two spectral features, one from each element that we are interested in, and compare the individual ratios from each star in our sample to identify any anomalous ratios. This is discussed in more detail in the above proceeding

Anderson: Given the spectroscopic variability you mentioned, have you considered photometric time-series from the EROS, OGLE, and MACHO surveys? Doing so could help find additional differences to RSGs, semi-regular variables, and Miras.

LEVESQUE: This star has been included in a number of different optical and IR surveys and has long been identified as a photometric variable; we haven't yet examined this data in detail but agree that it would be an interesting approach for potentially identifying other similar TŻO candidates in the future and better understanding HV 2112 in particular.

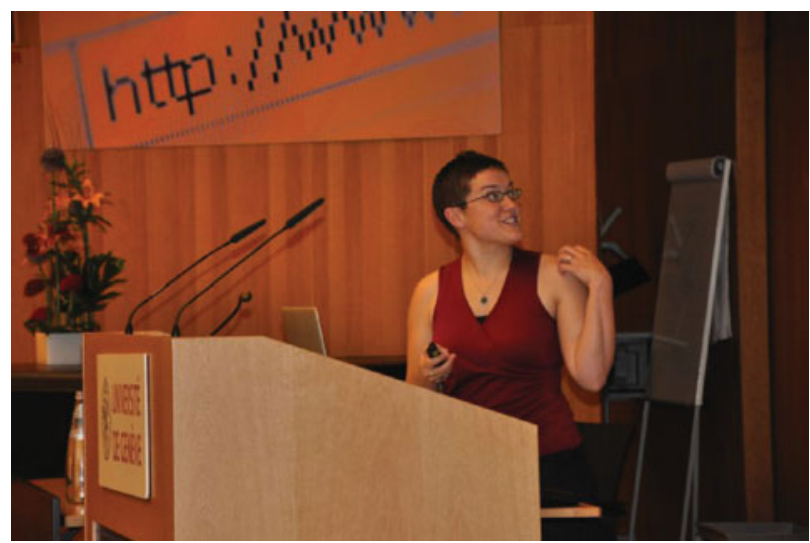

Emily Levesque

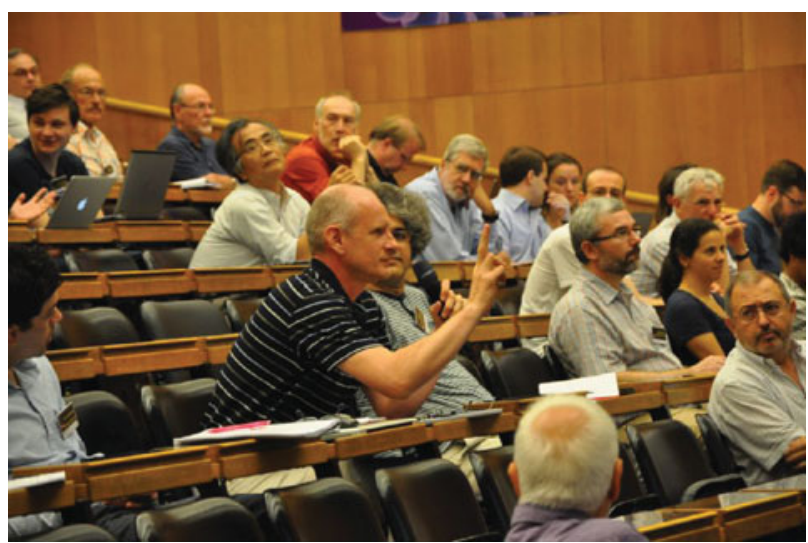

Alex de Koter asking a question 